\title{
KLF2 keeps B cells in their place
}

In this study, Hoek et al. describe a role for the transcription factor Kruppel-like factor 2 (KLF2) in the regulation of migratory receptor expression by marginal zone and follicular B cells. The authors show that the loss of KLF2 expression in B cells results in the disrupted positioning of these cells in the spleen and allows follicular B cells to respond to marginal zone-associated antigens.

Marginal zone B cells are noncirculating cells that reside in the white pulp of the spleen and mount responses to antigens without the need for T cell help. By contrast, follicular B cells are circulating cells and need to be localized near $\mathrm{T}$ cell areas to mount antigen-specific responses. KLF2 is involved in naive T cell trafficking, so the authors generated mice that specifically lacked Klf2 expression in B cells ( $K l f 2^{f l / f l} \mathrm{Cd} 19-\mathrm{Cre}^{+/-}$mice) to determine whether this transcription factor also has a role in $B$ cell migration. B cells in these mice prematurely exited the bone marrow, and there were higher numbers of both marginal zone and follicular B cells in the spleen compared with control mice. Of note, the marginal zone, but not the follicular area, was increased in size.
KLF2-deficient marginal zone B cells expressed lower levels of the migratory receptors $\mathrm{CXC}$ chemokine receptor 5 (CXCR5) and sphingosine 1-phosphate receptor 1 (S1P1). By contrast, KLF2-deficient follicular B cells expressed higher levels of these receptors and had enhanced migration in response to CXCR5 and S1P1 ligands compared with wild-type follicular B cells. Further analysis showed that follicular B cells were present in the marginal zone of $\mathrm{Klf} 2^{f l / f l} \mathrm{Cd} 19-\mathrm{Cre}^{+/-}$ mice, which indicates that disrupted $B$ cell trafficking occurs in the absence of KLF2.

Humoral immune responses to the T cell-independent type 2 antigen trinitrophenol (TNP)coupled Ficoll were enhanced in Klf $2^{f l / f l} \mathrm{Cd} 19-\mathrm{Cre}^{+/-}$mice. This antigen preferentially activates marginal zone $\mathrm{B}$ cells in wild-type mice; however, the enhanced response seen in Klf $2^{f l f l} \mathrm{Cd} 19-\mathrm{Cre}^{+/-}$mice was due to the defective homing of follicular $B$ cells into the marginal zone, as it was $\mathrm{T}$ cell dependent, required $\mathrm{S} 1 \mathrm{P} 1$, was associated with germinal centre formation and occurred in the absence of marginal zone B cells.
Finally, infection of $\mathrm{Klf} 2^{\mathrm{fl} / f l} \mathrm{Cd} 19-\mathrm{Cre}^{+/-}$ mice with Borrelia burgdorferi (the initial humoral response to which is dependent on marginal zone B cells) resulted in enhanced bacterial clearance and higher levels of IgG1 than in control mice. KLF2-deficient follicular B cells were shown to mediate this enhanced humoral response, which indicates that in the absence of KLF2, follicular B cells can respond to marginal zone-associated antigens.

So, KLF2 controls B cell localization in the spleen and prevents follicular B cells from gaining access to the marginal zone, thereby preventing follicular B cell responses to marginal zone antigens. This study raises a key question: why has the immune system evolved KLF2-dependent homing mechanisms that seem to be counterproductive to the clearance of blood-borne pathogens?

Olive Leavy

ORIGINAL RESEARCH PAPER Hoek, K. L. et al. Follicular $B$ cell trafficking within the spleen actively restricts humoral immune responses. Immunity 5 Aug 2010 (doi:10.1016/ j.immuni.2010.07.016) 